\title{
Evaluation of Alpha Glucosidase Inhibitor (Miglitol) for its Efficacy in Constipation Associated with Diabetes
}

\author{
Yogesh Kumar, Ajit Kumar Thakur*, Ramesh Kishorilal Goyal \\ School of Pharmaceutical Sciences, Delhi Pharmaceutical Sciences and Research University, M.B. Road, Pushp Vihar Sector-III, New \\ Delhi, INDIA.
}

\begin{abstract}
Objectives: Recently, miglitol emerged as effective $\alpha$-glucosidase inhibitor for the treatment of diabetic mellitus with specific advantages over currently available class of antidiabetic drugs. The aim of the present study was to evaluate the effects of miglitol in diabetes and associated constipation. Methods: Diabetes was induced in Wistar albino rat by streptozotocin given at dose of $25 \mathrm{mg} / \mathrm{kg}$, i.p. for 3 days consecutively to develop diabetes complications during next four weeks. The drugs were administered for 4 weeks starting from day 29 of post streptozotocin injections. Food and water intake, fecal parameters were measured on weekly basis. Gastrointestinal transit was monitored by charcoal meal and $\alpha$ - amylase activity was measured in all the treatment groups. Key findings: Treatment with miglitol demonstrated markedly increase in the gastrointestinal transit ratio in the diabetic rats and showed less $\alpha$ - amylase inhibitory activity, which providing evidences that miglitol does not exert constipation in the rats as compared to acarbose, which strongly inhibits the $\alpha$ - amylase enzyme and cause constipation in diabetic rats. Conclusion: These results suggest that miglitol has a potential to be used in the treatment of diabetes and associated constipation.
\end{abstract}

Key words: Miglitol, Diabetes, Constipation, Gastrointestinal motility, $\alpha$ - amylase enzyme activity.

\section{INTRODUCTION}

Constipation is one of the most common gastrointestinal symptoms in patients with diabetes $^{1}$ and occurs more frequently than in healthy individuals. Slow transit constipation, which is typically observed in diabetics and need to be controlled by proper diagnostics and treatment. ${ }^{2}$ Though there are various approaches to reduce the ill effects of diabetes and its associated complications. Recently, miglitol emerged as effective $\alpha$-glucosidase inhibitor for the treatment of diabetic mellitus with specific advantages over currently available class of antidiabetic drugs. Further it may produce flatulence, abdominal distention and cannot be used in individual with inflammatory bowel disease, gastroparesis. Miglitol is an $\alpha$-Glucosidase inhibitor $(\alpha-G I)$ with unique pharmacokinetic properties. $\alpha$-Glucosidase inhibitor competitively inhibits the absorption of $\alpha$-GI along the brush border of the small intestine which slow gut absorption of carbohydrate and ultimately reduces post prandial glucose. ${ }^{3}$ In some studies, $\alpha$-GI has also been shown to reduce post prandial hypertriglyceridemia. ${ }^{4}$ Miglitol also activates duodenal enterochromaffin cells, possibly by via Sodium-glucose transporter type 3 (SGLT-3) and mediates Glucagon-like peptide-1 (GLP-1) secretion through the parasympathetic nervous system. ${ }^{5}$ According to clinical study, miglitol suppresses the postprandial increase in interleukin 6 and enhances active glucagon-like peptide 1 secretion in viscerally obese subject. ${ }^{6} \mathrm{It}$ is previously reported that administration of
Submission Date: 15-12-2018; Revision Date: 06-02-2019; Accepted Date: 25-03-2019

DOI: 10.5530/ijper.53.3s.110 Correspondence: Dr. Ajit Kumar Thakur School of Pharmaceutical Sciences, Delhi Pharmaceutical Sciences and Research University (Govt. of NCT of Delhi), M.B. Road, Pushp Vihar Sector-III- 110017, New Delhi, INDIA. Phone: +91-11-29553481 E-mail: ajit.thakur@dpsru. edu.in

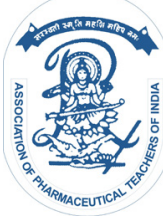

www.ijper.org 
$\alpha$-GI after meals improved compliance. ${ }^{7}$ One of the reasons for the poor compliance with $\alpha$-GI treatment is the incidence of adverse gastrointestinal effects. Since the adverse gastrointestinal effects of acarbose and miglitol have not been previously reported, in the present study we evaluated the effects of miglitol in diabetes and associated complications in gastrointestinal tract.

\section{MATERIALS AND METHODS}

\section{Animals and materials}

Wistar Albino rats (150-200g) of either sex was obtained from animal house, Delhi Pharmaceutical Sciences and Research University, New Delhi, India. The Experimental animals was approved by the Institutional Animal Ethical Committee (Protocol No: IAEC/2017-II/Prot. No. R-1). Principles of laboratory animal care $(\mathrm{NIH}$ publication number 85-23, revised in 1985) guidelines always followed. Miglitol (Glenmark, India), acarbose (Bayer, Brazil), streptozotocin (CDH, New Delhi) were procured. Other chemicals used in this study were analytical grade and purchased from authorized vendor of the University.

\section{Preparation of solution}

Accurately weigh amount of streptozotocin was dissolved in citrate buffer and $\mathrm{pH}$ was adjusted to 4.5 in less than one minute. The solutions were prepared fresh each time.

\section{Induction of diabetes and experimental design}

Diabetes was induced in rat by streptozotocin given at dose of $(25 \mathrm{mg} / \mathrm{kg}$, i.p.) for 3 consecutive days by using 25 gauze (5/8" long) needle in all groups except (Group I) which was normal control. ${ }^{8}$ After $72 \mathrm{~h}$ of streptozotocin injection, blood glucose level was estimated by glucometer (ACCU-CHEK, Roche Diabetes Care India Pvt. Ltd.). The animals showing blood glucose level greater than $180 \mathrm{mg} / \mathrm{dl}$ were considered as diabetic. The rats were allowed to develop diabetes complications for next four weeks. The animals were given standard rat pellet and tap water ad libitum.

Rats were randomly allotted to different experimental groups, each containing six animals $(n=6)$. Group I: Normal control; Group II: Diabetic control; Group III: Diabetic + Miglitol (100 mg/kg, p.o.); Group IV: Diabetic + Miglitol (200 mg/kg, p.o.); and Group V: Diabetic + Acarbose (100 mg/kg, p.o.). Miglitol and acarbose were administered for next 4 weeks starting from 5 th week of streptozotocin injection. After 8 weeks, blood samples were collected by cardiac puncture from deeply anesthetized rats. After collection of blood samples, rats were sacrificed for further study. Plasma was sep- arated by centrifuge 2200-2500 rpm (REMI C-24DL, Remi Elektrotechnik Ltd., Mumbai) for $10 \mathrm{~min}$, stored at $-20^{\circ} \mathrm{C}$ for enzyme estimation and small intestine track was excited for gastrointestinal parameters study.

\section{Food and water content}

Evaluation of food and water content that consumed by the rat were measured daily throughout the experiment with the help of electrical balance and a measuring cylinder.

\section{Fecal water content}

Each rat fecal were collected in the ceramic cups. Then wet weight of fecal pellets was determined. The water content was evaluated by drying the pellets in the oven at $70^{\circ} \mathrm{C}$ for $24 \mathrm{hr}$ and calculating the weight difference before and after drying. ${ }^{9}$ The water content was measured as follows:

Fecal water content $(\%)=[($ fecal wet weight - fecal dry weight $) /$ fecal wet weight $] \times 100$

\section{Determination of gastrointestinal transit}

All the animals fasted prior to the experiment for $16 \mathrm{hr}$ but consumed water ad libitum. After $15 \mathrm{~min}$ of administration of the drugs as per the experimental design, all the groups of animals received $0.5 \mathrm{ml}$ of charcoal meal $(10 \%$ charcoal in $5 \%$ gum arabic) through stomach tube. All the animals were sacrificed after $30 \mathrm{~min}$ of charcoal meal administration the intestinal tract of rats was removed to measure the travelled distance by the charcoal meal from the pylorus and expressed as a percentage of the total length of small intestine from gastro-pyloric junction to the ileocecal junction. ${ }^{9}$

$\mathrm{T}(\%)=\mathrm{B} / \mathrm{A} \times 100$

Where $\mathrm{T}$ is the intestinal tract motility percent, $\mathrm{A}$ is the total length of the intestinal tract and B is the travelled distance of the most distal end portion by the charcoal.

\section{Alpha amylase enzymatic assay}

The $\alpha$-amylase is an enzyme that hydrolyses $\alpha$-bond of polysaccharides, such as starch and glycogen, yielding glucose and maltose. The $\alpha$ - amylase enzyme activity was measured by the method published elsewhere.

\section{Method}

$\alpha$ - amylase inhibitory activity was carried out according to the standard method with minor modification. ${ }^{10}$ Reaction mixture containing buffer solution $(20 \mathrm{mM}$ sodium phosphate with $6.7 \mathrm{mM}$ sodium chloride, $\mathrm{pH}$ 6.9 at $\left.20^{\circ} \mathrm{C}\right), 100 \mu \mathrm{l}$ of blood plasma was preincubated at $20^{\circ} \mathrm{C}$ for $3 \mathrm{~min}$. Then, the $(1 \mathrm{ml})$ starch solution $[1 \%$ $(\mathrm{w} / \mathrm{v})$ soluble starch solution] was added as a substrate and incubated further at $20^{\circ} \mathrm{C}$ for $3 \mathrm{~min} ;(1 \mathrm{ml})$ of DNS 
color reagent was then added and boiled for exactly 15 min. The absorbance of the resulting was measured at $540 \mathrm{~nm}$ using UV-Visible spectrophotometer (UV-1800, Shimadzu, Japan). Alpha amylase enzymatic assay was performed in triplicates method. The results of enzymatic assay were expressed as percentage inhibition, which was calculated using the formula.

$\%$ inhibition $=($ Absorbance $($ control $)-$ Absorbance (test)) $/$ (Absorbance $($ control $)) \times 100$

\section{Statistical analysis}

All data are expressed in mean $\pm \mathrm{SD}(n=6)$. Differences between the groups were compared by one-way ANOVA followed by Newman- Keuls test using the Graph Pad Prism Version 5 software. All the observational value $P<0.05$ was always considered statistically significant.

\section{RESULTS}

\section{Effects of miglitol and acarbose on food and water intake}

The results of miglitol and acarbose on food and water intake were statistical insignificant $(P>0.05)$. Whereas groups treated with miglitol at dose of (100 and 200 $\mathrm{mg} / \mathrm{kg}$ ) did not show significant changes in food intake or water consumption. Standard drug acarbose groups also did not change food intake or water consumption.

\section{Fecal parameter}

The water content was decreased in the groups treated with miglitol at doses of (100 and $200 \mathrm{mg} / \mathrm{kg}$ ) as compared to diabetic control group and groups treated by standard drug acarbose was also showed decreased in water content as compared to diabetic control groups (Figure 1a).

\section{Gastrointestinal motility}

Gastrointestinal transit ratio of diabetic control rats after 8 weeks of study was found to be significantly $(P<0.05)$ decreased and at the same duration of study gastrointestinal transit ratio of group treated with miglitol orally at (100 and $200 \mathrm{mg} / \mathrm{kg}$ ) were significantly $(P<$ $0.05)$ increased whereas in acarbose (standard) group also showed increased in intestinal transit ratio (Figure 1b).

\section{Alpha amylase enzymatic assay}

Miglitol treated rats at doses of (100 and $200 \mathrm{mg} / \mathrm{kg}$ ) showed less inhibitory activity of $\alpha$-amylase whereas the acarbose treated rats showed greater inhibitory activity of $\alpha$-amylase as compared to miglitol groups (Table 1).

\begin{tabular}{|c|c|}
\hline Groups & Enzyme inhibition (\%) \\
\hline Normal control & - \\
\hline Diabetic control & $40.30 \pm 1.98$ \\
\hline Diabetic + miglitol $(100$ mg/kg) & $51.64 \pm 0.53$ \\
\hline Diabetic + miglitol $(200$ mg/kg) & $51.10 \pm 0.65$ \\
\hline Diabetic + acarbose $(200 \mathrm{mg} / \mathrm{kg})$ & $86.78 \pm 1.10$ \\
\hline
\end{tabular}

(a)

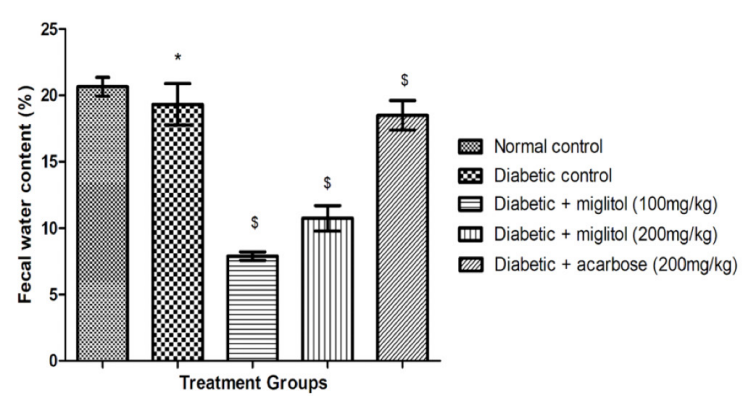

(b)

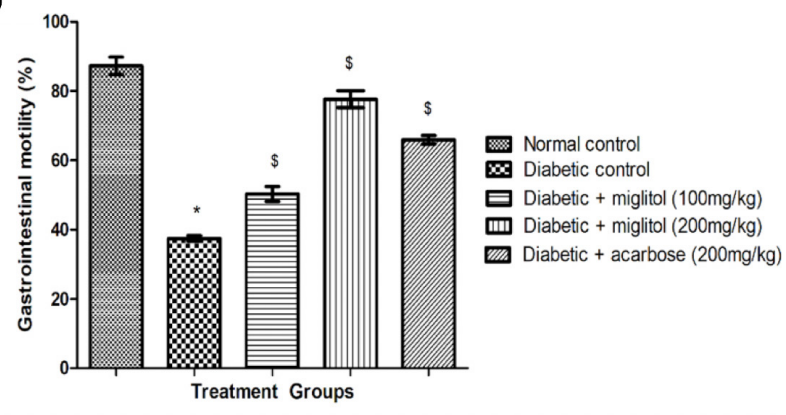

Figure 1: Effect of drugs treatment on fecal water content (a) and on gastrointestinal motili-ty (b). ${ }^{*} p<0.05$ versus normal control; $\$<0.05$ versus diabetic control.

\section{DISCUSSION}

Gastroparesis and intestinal enteropathy are gastrointestinal symptoms mainly caused by diabetes. Intestinal enteropathy can cause diarrhea, constipation and fecal incontinence. Patients with diabetes may have neuropathy that affects gastrointestinal motility. Diabetes caused smooth muscle myopathy which impairs the gastrointestinal motility and results in delayed gastrointestinal transit time. The particular mechanisms involved in the formation of constipation in patients with diabetes are not completely understood. In the present study we evaluated the effects of miglitol in diabetes and associated complications in gastrointestinal tract. However, in this study results of food intake and water consumption were insignificant $(P>0.05)$. Whereas groups treated with miglitol did not show significant changes in food intake or water consumption. Standard drug acarbose groups also did not change food intake or water 
consumption. All above results indicate that diabetes induced was moderate like Type 2. It is because the dose used was less. Normally, in most studies (45 mg/ $\mathrm{kg}$ i.p.) dose is used for diabetes mellitus. We examined the effects of miglitol and acarbose on gastrointestinal transit as well as related disorders. Fecal parameters such as fecal water content have been used as indices of the effect of the drugs. In the present study, streptozotocin induced diabetes in rats showed higher fecal water content. After the treatment with miglitol and acarbose the fecal water content was decreased as compared to diabetic control group.

The transit process of the whole gastrointestinal tracts shows the overall gastrointestinal motor activity. In this manner, estimating gastrointestinal charcoal transit ratio is additionally helpful in the finding of constipation. A decreased in the gastrointestinal charcoal transit ratio demonstrates constipation. ${ }^{11,12}$ In the present study, significant decrease in the gastrointestinal charcoal transit ratio was seen in diabetic control rats compared to normal rats. The groups treated with miglitol and acarbose showed increased gastrointestinal charcoal transit ratio as compared to diabetic control rats, indicates that they do not exert constipation in the rats. However, the effects of miglitol were better than acarbose in the gastrointestinal motility.

The $\alpha$ - amylase inhibitory studies performed to demonstrate the effects of miglitol and acarbose on the $\alpha$ - amylase enzyme. This enzyme is most prominent in pancreatic juice and saliva. Inhibition of $\alpha$-amylase enzymes which is involved in the digestion of carbohydrates can significantly decrease the elevated postprandial blood glucose after a mixed carbohydrates diet. Therefore, inhibition of $\alpha$-amylase can be an important strategy in the management of postprandial blood glucose level in type 2 diabetic patients. ${ }^{13}$ According to the above findings, this study suggested that diabetic group showed less $\alpha$ - amylase inhibitory activity and treatment with miglitol showed some $\alpha$ - amylase inhibitory activity whereas treatment with acarbose strongly inhibits $\alpha$ - amylase. Therefore, the administration of miglitol increased unabsorbed disaccharides, rather than polysaccharides, in the intestine.

\section{CONCLUSION}

Our observational results suggest that miglitol is effective in the diabetes induced constipation. Results of miglitol were comparable with acarbose. Miglitol also appears to be effective in prevention of diabetes induced gastrointestinal disturbances like constipation. There may be independent of $\alpha$-amylase enzyme, as similar effects were not observed with acarbose. Further, effects of miglitol in treatment of diabetes were better than acarbose.

\section{ACKNOWLEDGEMENT}

This work was supported by Research funding to Delhi Pharmaceutical Sciences and Research University from Govt. of NCT of Delhi.

\section{CONFLICT OF INTEREST}

The authors declare no conflict of interest.

\section{ABBREVIATIONS}

$\alpha$-GI: $\alpha$-Glucosidase inhibitor; SGLT-3: Sodium-glucose transporter type 3; GLP-1: Glucagon-like peptide-1.

\section{REFERENCES}

1. Prasad VG, Abraham P. Management of chronic constipation in patients with diabetes mellitus. Indian J Gastroenterol. 2017;36(1):11-22.

2. Rossol S. Constipation in patients with diabetes mellitus. MMW Fortschr Med. 2007;149(44):39-42.

3. Inzucchi SE, Bergenstal RM, Buse JB, Diamant M, Ferrannini E, Nauck M, et al. Management of Hyperglycemia in Type 2 Diabetes: A Patient- Centered Approach. Diabetes Care. 2012;35(6):1364-79.

4. Hariya N, Mochizuki K, Inoue S, Saito M, Fuchigami M, Goda T, et al. Switching a-Glucosidase Inhibitors to Miglitol Reduced Glucose Fluctuations and Circulating Cardiovascular Disease Risk Factors in Type 2 Diabetic Japanese Patients. Drugs R D. 2014;14(3):177-84.

5. Woodman RJ, Mori TA, Burke V, Puddey IB, Watts GF, Beilin LJ. Effects of purified eicosapentaenoic and docosahexaenoic acids on glycemic control, blood pressure and serum lipids in type 2 diabetic patients with treated hypertension. Am J Clin Nutr. 2002;76(5):1007-15.

6. Arakawa M, Ebato C, Mita T, Fujitani Y, Shimizu T, Watada H, et al. Miglitol suppresses the postprandial increase in interleukin 6 and enhances active glucagon-like peptide 1 secretion in viscerally obese subjects. Metabolism. 2008;57(9):1299-306.

7. Aoki K, Nakamura A, Ito S, Nezu U, Iwasaki T, Takahashi M, et al. Administration of miglitol until 30 min after the start of a meal is effective in type 2 diabetic patients. Diabetes Res Clin Pract. 2007;78(1):30-3.

8. Xiang X, Wang Z, Zhu Y, Bian L, Yang Y. Dosage of streptozocin in inducing rat model of type 2 diabetes mellitus. Wei Sheng Yan Jiu. 2010;39(2):138-42.

9. Yu LL, Liao JF, Chen CF. Anti-diarrheal effect of water extract of Evodiae fructus in mice. J Ethanopharmacol. 2000;73(1-2):39-45.

10. Ademiluyi AO, Oboh G. Soybean phenolic-rich extracts inhibit keyenzymes linked to type 2 diabetes ( $\alpha$-amylase and a-glucosidase) and hypertension (angiotensin I converting enzyme) in vitro. Exp Toxicol Pathol. 2013;65(3):305-9.

11. Wintola OA, Sunmonu TO, Afolayan AJ. The effect of Aloe ferox Mill. in the treatment of loperamide induced constipation in Wistar rats. BMC Gastroenterol. 2010;10(1):95. doi: 10.1186/1471-230X-10-95.

12. Sagar L, Sehgal R, Ojha S. Evaluation of antimotility effect of Lantana camara L. var. acuelata constituents on neostigmine induced gastrointestinal transit in mice. BMC Complement Altern Med. 2005;5(1):18. doi.org/10.1186/14726882-5-18.

13. Tiwari AK, Rao JM. Diabetes mellitus and multiple therapeutic approaches of phytochemicals: Present status and future prospects. Curr Sci. 2002;83(1):30-8. 


\section{PICTORIAL ABSTRACT}

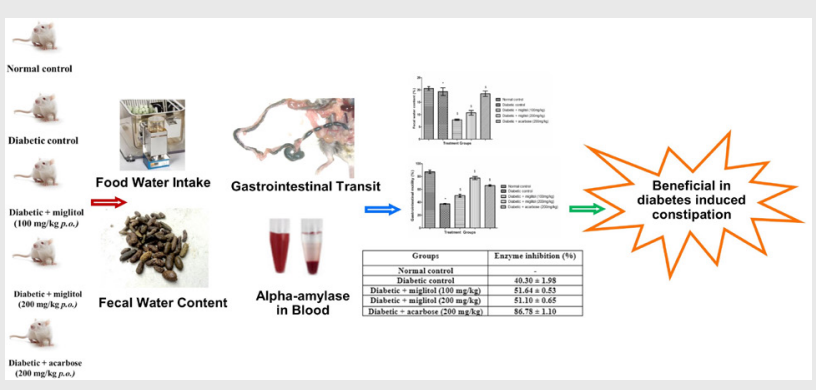

\section{SUMMARY}

Miglitol emerged has effective $\alpha$-glucosidase inhibitor for the treatment of diabetic mellitus. Present findings demonstrate that miglitol as a potential to be used in the treatment of diabetes and associated constipation. Further biomarker-based study are warranted for proper understanding the mechanism of action(s).

\section{About Authors}

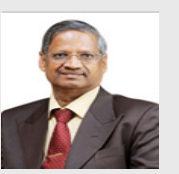

Prof. Ramesh Kishorilal Goyal: Vice-chancellor of Delhi Pharmaceutical Sciences and Research University, New Delhi, India.

Dr. Ajit Kumar Thakur: Assistant Professor of Pharmacology at School of Pharmaceutical Sciences, Delhi Pharmaceutical Sciences and Research University, New Delhi, India.

Mr. Yogesh Kumar: M.Pharm student at School of Pharmaceutical Sciences, Delhi Pharmaceutical Sciences and Research University, New Delhi, India.

Cite this article: Kumar Y, Thakur AK, Goyal RK. Evaluation of Alpha Glucosidase Inhibitor (Miglitol) for its Efficacy in Constipation Associated with Diabetes. Indian J of Pharmaceutical Education and Research. 2019;53(3 Suppl 2):s387-s391. 